\title{
La productivité des ratures dans le travail de Ferdinand de Saussure ${ }^{1}$
}

\author{
Silveira, Eliane \\ Universidade Federal de Uberlândia/Brasil - CNPq \\ eliane.m.silveira@gmail.com
}

\section{Introduction}

Saussure reste une énigme pour beaucoup de personnes, des linguistes surtout mais aussi pour quelques philosophes et psychanalystes. Cette énigme réapparaît tout au long des temps, animée par des causes différentes - connues ou présumées - et en suscite d'autres, avec ou sans raison. Cependant, cela importe moins que le fait que la production de Saussure continue à être la raison de divers travaux.

Dans la Linguistique en particulier, ce qui avait été révolutionnaire à un moment donné est devenu d'un côté discrédité, d'un autre, figé: les critiques aux exclusions lues dans les dichotomies saussuriennes langue vs parole, synchronie vs diachronie - s'intensifient et, surtout, le Cours de Linguistique Générale devient une lecture obligatoire à l'Université, mais souvent en ayant le statut de lettre morte, sans aucun rapport avec la théorisation sur le langage, tout en restant comme une simple information pour déterminer sa différence par rapport à tant d'autres théories linguistiques.

En outre, l'œuvre de Ferdinand de Saussure, maints auteurs l'ont déjà écrit, demeure intouchée. Il n'a pas écrit le Cours de Linguistique Générale. Cet ouvrage est le fruit d'une édition construite à partir de quelques-uns de ses manuscrits et des notes de ses élèves. Cela suscite, actuellement, des discussions très vives de la part des chercheurs qui sont partagés dans leur jugement du travail des éditeurs. De plus, les cours ayant donné naissance aux notes qui ont fourni la matière de cette édition ne résument pas la production du linguiste genevois. Il a écrit près de dix mille feuilles dont la plupart se trouvent à présent archivées à la Bibliothèque Publique de Genève et une petite partie à la Houghton Library de l'Université de Harvard.

Le travail sur ces manuscrits a engendré, au départ, une interprétation de la production du linguiste comme une dichotomie: le 'Saussure diurne', celui des cours qui sont à l'origine du Cours de Linguistique Générale et le 'Saussure nocturne', révélé par quelques manuscrits. L'obscur Saussure nocturne est privé de lumière, n'ayant donc pas assez d'éclairage à sa compréhension. L'importance des différents travaux de Saussure semblait aller au-delà de cette dichotomie.

Saussure, lui-même, reconnaît sa difficulté aussi bien à traiter les anagrammes qu'à les abandonner: “(...) Je ne sais si c'est par inspiration des tombeaux des Scipions ou autrement que j'ai passé ensuite mon temps à [traiter] creuser le vers saturnien (...)". (Saussure, apud Starobinski, p.30). Starobinski dira : "Conviction et doute" (p. 90) Il y a des gênes dans ses lettres sur ses découvertes - Il ne s'agit pas d'une certitude délirante sur sa découverte - ce n'est pas de la folie - Il s'agit de hasard? Saussure dira également: "Pourquoi une lettre aurait-elle été alors désignée par une baguette? Mystère." (Saussure, apud Starobinski, p.30)

Mais, les manuscrits, au-delà des anagrammes, offrent beaucoup de mystères et à propos des spécificités du manuscrit saussurien, Starobinski lui-même sera amené à prendre position devant un des aspects importants de ces manuscrits :. «Pour ne pas en compliquer la lecture, nous ne nous occuperons pas, mais seulement à titre exceptionnel, des termes raturés. » (p 8/9)

Godel (1957) avait déjà écrit:

Les additions en marge et les retouches y sont fréquentes; souvent la phrase, plus d'une fois recommencée, reste en suspens; un mot, parfois un mot essentiel, est 
laissé en blanc, par endroits, l'ordre même du texte ne se révèle qu'à une recherche patiente. (op.cit:36).

Parret (1995), en se rapportant aux manuscrits de Harvard, a écrit:

Il m'est impossible de traiter exhaustivement tous ces matériaux - je manque de connaissances techniques pour embrasser l'ensemble des domaines couverts et des perspectives ouvertes par les manuscrits de Harvard." Il dira en outre que "Tout un champ d'inquiétudes est ainsi suggéré dans les manuscrits de Harvard[...] (op.cit.:13)

en y ajoutant une affirmation qui côtoie celle de Godel, citée ci-dessus:

Mais les notes inédites (de 1954), les Sources manuscrites tout comme les fragments très importants du Fascicule 4 n'offrent précisément qu'un canevas, ce qui nous force de combler les vides, à rendre explicite ce qui n'est que suggestif, de résoudre les contradictions, voire les paradoxes. (op.cit:19)

Les manuscrits sont très intéressants dans ce sens et remplis de phrases interrompues avant leur fin, de mots répétés exhaustivement et signalés par d'innombrables ratures.

Ces espaces qui, dans un manuscrit sont voués au non-sens, peuvent être intéressants à certains égards. Normand réalise une réflexion quant aux espaces vides dans les manuscrits saussuriens de façon à indiquer en eux une fonction.

Lors d'une conférence en 2003, en mémoire à Rudolf Engler, Claudine Normand s'est proposée de travailler sur le «blanc» qui la fascinait dans les manuscrits saussuriens ; ainsi est apparu le texte «Les espaces vides ( ou les espaces en blanc. ou les blancs) dans les manuscrits saussuriens » dans lequel l'auteure, en ses propres mots, se propose de « voir dans ces blancs de la voix, ces interruptions du geste de la main, la présence insistante du corps qui ne se laisse pas facilement chasser de la réflexion théorique. » (2006, p. 82). Normand se rend compte que les éditions critiques ne prennent pas les blancs de manière précise et systématique et pour cela ne semblent pas significatifs, sont considérés comme des ratures, appelés lacunes, absences et vides, Elle choisit de les appeler blancs pour « accentuer à la différence de la visibilité, la matérialité spécifique de leur présence dans le texte ». (op. cit., p. 83)

Alors, à partir du choix de travailler avec certains éléments qui tendent à montrer la précarité du sens dans la langue, dans ce travail nous signalerons, dans quelques pages de ses notes pour la Première Conférence $^{2}$ (1891), le mouvement tortueux du linguiste, en quête de définition de la Linguistique en tant que science, à travers les ratures, les incises et les répétitions présentes dans ce manuscrit, ce qui nous apparaît, maintenant, comme le 'point aveugle' dans les manuscrits saussuriens.

Ce travail, est centré surtout sur une analyse des manuscrits de Ferdinand de Saussure: Première Conférence à l'Université (cours d'ouverture) qui contient 30 feuilles, localisées et cédées par $\mathbf{M}^{\mathrm{me}}$ Marie de Saussure et organisées, classées et archivées dans la Bibliothèque Publique de Genève, par Robert Godel. Cette première conférence, objet de notre intérêt, fait partie d'un ensemble plus grand de manuscrits qui ont servi comme notes préparatoires pour les trois premières conférences de Saussure ${ }^{3}$ à Genève et marque son retour à sa terre natale.

Notre analyse se concentre sur les moments d'interruption du lisible provoqués par les hésitations de Saussure lors de l'écriture de son texte et met l'enjeu sur la productivité de telles hésitations. En outre cette première conférence mérite notre attention sur la façon avec laquelle Saussure a cherché à y construire un discours à propos de ce qu'est la linguistique et de ce qu'est le rôle du linguiste à partir de la nature de son objet, qui ne lui paraît pas, non plus, éclairée.

Notre analyse nous a permis de différencier trois groupes de ratures à partir des thèmes délimités par eux-mêmes. Le thème du premier groupe semble être cher à Saussure: la place des études du langage dans le cadre des sciences humaines. Le thème du deuxième groupe est très cher à la linguistique ellemême: la nature de son objet. Dans le troisième groupe, nous trouvons les questions qui concernent le 
général et le particulier dans l'approche de l'objet de la linguistique et finalement, ce qui semble être demeuré dans l'ombre d'une élaboration dans ce manuscrit: la parole.

L'analyse de ces trois groupes de ratures, qui gravitent autour de certains thèmes, nous a permis de montrer des moments de tension et/ou d'hésitation lors de l'élaboration des concepts qui devaient encore être définis, plus spécifiquement ce qui se présente est la nécessité de définir ce qui a toujours semblé si imprécis à Saussure: la position du linguiste devant le langage et devant la linguistique en tant que science. L'analyse nous a aussi permis d'entrevoir des solutions à ces questions-là ainsi que de reconnaître un point sur lequel une question insiste, sans pour autant avancer.

Les ratures que l'on a choisies semblent fonctionner comme le noyau d'une constellation constituée d'autres termes qui y gravitent autour et maintiennent avec lui des relations de signification; en outre, des ratures sont présentes sur les mêmes termes, ou sur des termes avec une signification semblable, et souvent sur la même feuille. Pourtant, sur les feuilles qui se suivent, les sens qui semblaient interrompus par la rature commencent à se formuler de façon précise à propos de chacun de ces thèmes que nous avons classés dans les trois groupes.

\section{2 "Trois premières conférences à l'Université (cours d'ouverture, nov.1891)"}

Initialement, nous présenterons l'ensemble des manuscrits où figure la Première Conférence à l'Université (cours d'ouverture), à la fois en ce qui concerne sa forme et son contenu. Pour le premier ensemble de manuscrits de la rubrique 3951 qui est justement «Trois premières conférences à l'Université (cours d'ouverture, nov. 1891) ». Il s'agit d'un document contenant trois ensembles de manuscrits, chacun d'entre eux nommés par Godel, comme $1^{\text {er }}$ Cours d'ouverture, $2^{\text {ème }}$ Cours d'ouverture, et le dernier comme $3^{\text {ème }}$ Cours d'ouverture. Après cette page de titrage, suit une sorte de résumé de sujets indiquant le numéro des pages où apparaît un certain contenu travaillé par Saussure dans chacun de ses manuscrits. À partir de là, nous avons les pages écrites par Saussure et celles écrites par Godel. Le premier manuscrit va jusqu'à la page 30 , le deuxième jusqu'à la page 13 et le troisième jusqu'à la page 22 (totalisant 65 pages ; néanmoins, il y a aussi les pages non numérotées dans les deuxième et troisième ensembles de manuscrits. Dans le deuxième ensemble, il y a une page avec un numéro répété (11 a) dont la longueur dépasse le format d'une feuille A4 et s'étend donc sur une autre page, de sorte que l'extension de la page 11 de ce manuscrit de Saussure est presque l'équivalent de trois pages format A4. Dans le troisième, il y a une page non numérotée dont la forme et le contenu de l'écriture indiquent que Saussure aurait commencé à écrire une première fois, aurait raturé, se serait arrêté et aurait recommencé, dans la même direction sur une autre feuille, numérotée page 6 par Godel. Comme nous l'avons déjà dit, nous nous attarderons seulement sur la Première Conférence à l'Université (cours d'ouverture) sur laquelle nous discourrons plus attentivement.

Lors de cette conférence comme le montre Godel et comme nous pouvons le constater, Saussure produit «Justification de la linguistique comme science autonome par son utilité pour d'autres sciences (philologie, histoire, etc.) et surtout par l'importance de son objet: le 'phénomène du langage." (op. cit.:37) Saussure affirmera aussi au cours de cette conférence que l'étude du langage comme fait humain est entièrement, où presque, l'étude des langues.

Saussure réitérera une position connue dans laquelle il défend que le langage n'est pas une science naturelle mais une science historique et montre la distinction nécessaire entre la langue dans l'histoire et l'histoire des langues ${ }^{4}$. Saussure traitera, comme l'affirme Godel, du « caractère exclusivement historique du langage et de la science du langage; il avait pourtant déjà acquis la conviction que la linguistique est une science double, et que la langue ne vit pas uniquement d'événements." (op.cit.:2627). À noter également sa justification des études linguistiques dans une faculté de Lettres. Par ailleurs, Saussure apporte ce qu'il appelle «degrés de la volonté » comme nous le dit Godel "Il faut d'abord distinguer la langue dans l'histoire et l'histoire des langues; mais, en outre, pour qu'une science soit une science historique, il faut que son objet représente des actes humains, réglés par la volonté et 
l'intelligence, et qui intéressent la collectivité. C'est le cas de la linguistique, pourvu que l'on distingue des degrés de la volonté (consciente ou inconsciente): l'acte linguistique est le moins réfléchi, le moins prémédité, le plus impersonnel de tous. Mais il n'y a là qu'une différence de degré (p.18)." (op.cit.:38)

Saussure traitera finalement dans cette conférence, toujours selon Godel, de la « diversité et de la continuité de la langue dans le temps et dans l'espace », et pour lui le parler humain se transmet sans interruption et même dans les cas appelés échanges de langue, ce qu'il y a effectivement est une illusion, ce sont des noms donnés à des états distincts de langue (latin, français, portugais).

L'étroite relation entre les élaborations de Saussure en 1891 et celles qui se présentent dans ses cours, deux décades plus tard, sont remarquées par De Mauro quand il affirme que le troisième cours va précisément dans cette direction: il intègre l'ordre déductif du second cours à la richesse analytique du premier. Il développe, au début, le thème "les langues", c'est-à-dire la linguistique externe. C'est là un vieux postulat de Saussure: déjà en 1891, dans les trois leçons d'ouverture aux cours genevois, il avait soutenu que 'les plus élémentaires des phénomènes du langage ne seront soupçonnés ou clairement aperçus, classés et compris si l'on ne recourt en première et dernière instance à l'étude des langues... D'un autre côté, vouloir étudier les langues en oubliant que ces langues sont primordialement régies par certains principes qui sont résumés dans l'idée du langage, est un travail encore plus dénué de toute signification sérieuse, de toute base scientifique véritable. Sans cesse, par conséquent, l'étude générale du langage s'alimentera des observations de toutes sortes qui auront été faites dans le champ particulier de telle ou telle langue...' (Notes 65) Une dialectique incessante lie l'étude générale et l'étude historicodescriptive: mais d'un point de vue didactique, le prius revient aux 'langues' car 'l'exercice de cette fonction [ le langage] n'est abordable que ... par le côté des langues existantes'(ibid).” (op.cit.:354). De Mauro note qu'il s'agit d'une dialectique incessante entre l'état général et l'état historico-descriptif.

Ces observations donnent une bonne idée du manuscrit, cependant elles ne donnent pas toute la dimension complète du parcours de Saussure dans son élaboration théorique, Saussure est immergé dans un travail qui peut être caractérisé, comme Normand l'a fait :

\begin{abstract}
L'étude philologique des blancs devrait certainement être plus systématique ; je ne crois pas cependant qu'elle puisse éclairer d'un jour nouveau les difficultés théoriques de Saussure. Ce que je retiendrai, personnellement, de cette intrusion dans l'intimité de ces écrits c'est un trait qui rapproche Saussure de la plupart d'entre nous, me semble-t-il: que la pensée n'émerge que par à coups, intermittences, d'un fond sonore confus où se mêlent les discours entendus, 'les vois chères qui se sont rues', toute une phraséologie ancienne. Le plus souvent c'est un bruit brouillé, que manifestent aussi les hésitations de la main, ratures et ajoutes, toutes traces de cette activité 'epilinguistique qu'Antoine Culioli pose à la source de toutes les verbalisations sans qu'elle puisse elle-même être directement verbalisée. Plus rêveusement j'aime penser que dans ces blancs saussuriens se concrétise le corps subtil dont rêvait Roland Barthes, l'utopie du 'bruissement de la langue'. (op.cit. p.91)
\end{abstract}

Dans ce travail, nous cherchons à rester attentifs aux destructions, ou c'est-à-dire à ce qui manque ou excède dans l'écriture du manuscrit, et cela parce que l'objectif est de situer la perturbation dans ce qui est déjà établi comme connaissances en linguistique et dont l'hésitation permet l'apparition d'une nouvelle élaboration, avec comme objectif, la forme de l'écriture (ratures, incises, interruptions et répétitions) qui devient fondamentale pour ce travail. À savoir, dans ce cas, spécifiquement, nous ne convoitons pas une édition critique ou même n'atteignons pas la genèse des concepts élaborés par Ferdinand de Saussure, bien que nous voulions fournir des éléments pour une lecture du manuscrit (tâche toujours bien atteinte par l'édition critique) et aussi que nous n'allions pas les laisser s'échapper quand dans ce manuscrit quelques éléments indiquent la genèse des théories de Saussure que nous connaissons actuellement. 


\section{Premier groupe: la science linguistique}

Les premiers mots du manuscrit Première Conférence à l'Université (cours d'ouverture) mentionnent déjà « un ordre de nouvelles études ». Cependant, on rencontre, ensuite, quelques mots raturés qui semblent être dirigés vers un questionnement de ce que l'on attend de ces nouvelles études : but, utilité et place. C'est là que l'on délimite notre premier groupe. Ces termes se répètent exhaustivement : place, sept fois et les deux autres mots, au moins trois fois. Parfois raturés, mais toujours indicatifs d'une question sur la position que les études du langage possèdent dans le cercle des connaissances humaines, spécialement dans une Faculté de Lettres. Ces termes se présentent d'une façon insistante et gênante comme on peut le voir dans les répétitions et les ratures de mots comme utilité, but et intérêt : "de vous introduire aujourd'hui??? entretenir du but, de l'utilité, de soit du but de ces études, soit de l'utilité de ces études, soit de leur intérêt".

De tels mots conservent un certain voisinage sémantique mais n'ont pas la même signification quand il s'agit de définir la fonction d'un domaine d'étude, principalement quand on discute la légitimité de ce domaine. Tous ces mots ont été mis dans des incises, répétés ou raturés en montrant une certaine gêne dans la qualification de 'ces études'. Après toutes ces hésitations précédentes, le mot légitimité est ajouté par Saussure aux mots but, intérêt et utilité dans un texte sans ratures : soit de l'utilité, soit de leur intérêt, soit enfin de leur but et de leur légitimité. On note que ce texte sans ratures répète, en mettant à nouveau en ordre, le matériel raturé comme si la dernière expression (leur légitimité) opérait une resignification nécessaire.

La gêne initiale semble être de l'ordre d'un dérangement avec le lieu qui occupait les études du langage et, aussi, elle indique un effort pour parler du lieu qu'ils pourraient occuper dans le cercle des connaissances humaines. Voici un premier déplacement.

Comme on l'a déjà montré avant, un des points qui signalisent le déplacement est l'utilisation des termes utilité et légitimité qui semblent être marqués par une incompatibilité quand il s'agit de caractériser une science, vu que l'utilité d'une étude peut légitimer une technique mais non une science.

Parmi quelques lignes raturées dans le manuscrit et l'incise à côté des ratures dans la première page, on comprend que quelque chose a été écrit, barré et, de plus, ajouté à ce qui pourrait être le remplaçant de ce qui avait été écrit initialement : et j'aurais en outre examiner quelle place et la place (...)elle doit өccuper. Et l'incise : à marquer la place qu'elle occupe dans le cercle(...). Le remplacement opéré dans ce mouvement est digne de note. Examiner passe à marquer et on doit dire que ces verbes aboutissent à des pratiques distinctes. 'Examiner' a le sens d'analyser avec attention et minutie, considérer, pondérer... tandis que 'marquer'est mettre une marque, un signal, un insigne ; donc, dans cet extrait du manuscrit on traite de signaler quelque chose déjà examiné. Tout cela nous montre que, tandis que l'un appartient à l'ordre de l'observation, l'autre appartient à l'ordre de l'action. De la rature à l'incise, on ébauche une position plus incisive devant ce qu'il y a encore à définir dans les études du langage.

Il est significatif qu'à la fin de la page qui contient ces ratures, on lise la question : quel est le profit que l'érudition classique peut retirer des études du langage et l'intérêt que la même étude peut avoir pour l'histoire ou pour l'histoire de la civilisation? Presque comme une réponse à cette question, il surgit dans le texte une série d'indications sur comment les études du langage ont été utiles pour la résolution de questions historiques et ethnologiques. Ainsi, bien qu'il n'y ait pas de reprise des mots du premier groupe, on peut affirmer qu'ils opèrent, en absence de celle-ci, dans cette élaboration une fois que, quand il s'agit de dire dans quels points l'étude du langage et ce qui est nommé 'sciences adjacentes' peuvent se toucher ou pas, ces mots du premier groupe ont une importance capitale et l'utilisation de termes comme profit et intérêt le confirme.

Donc, deux questions éclaircissant la direction contraire que le texte va prendre, surgissent dans les pages trois et quatre : l'utilité comme obstacle. Voyons : 


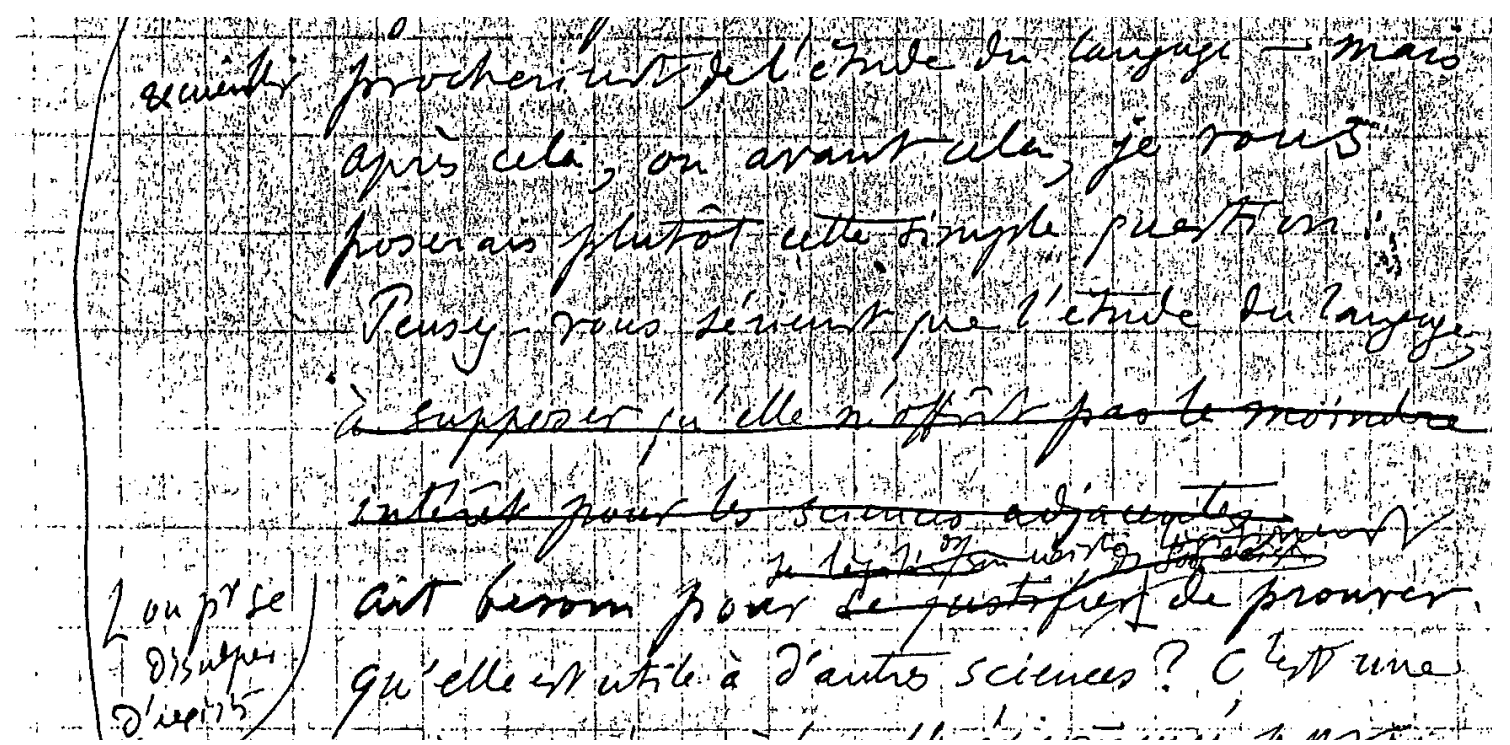

Et en plus:

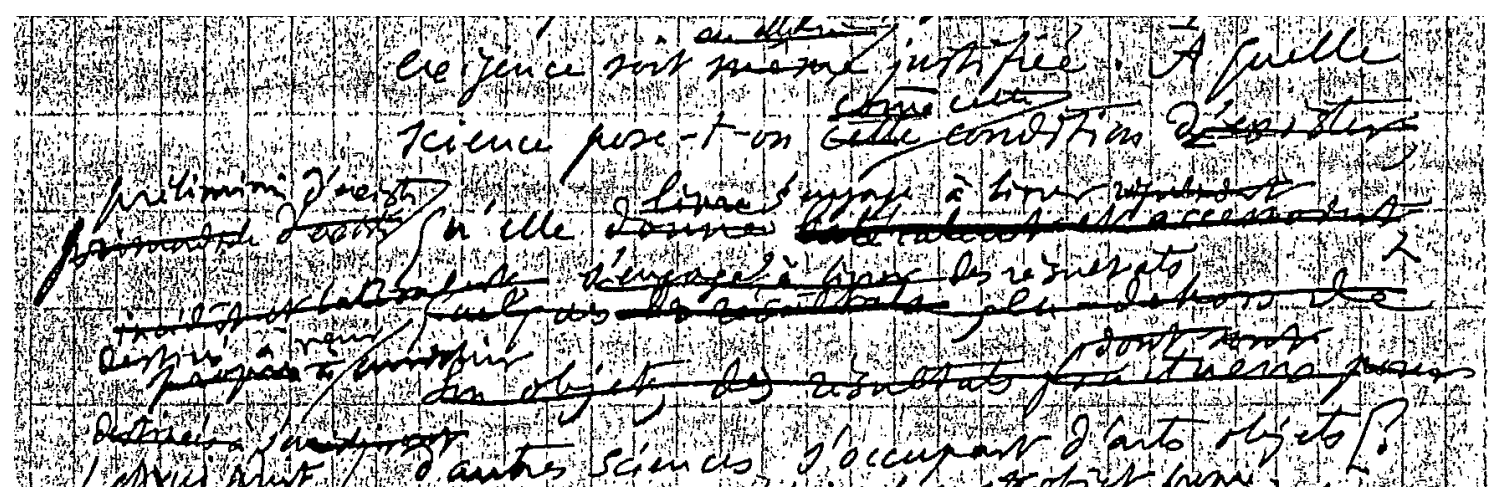

Ces questions montrent le besoin de séparer la linguistique de ces sciences, vu que les rapports entre elles sont marqués par but, utilité et profit. Donc, il faut savoir, d'après la page quatre du manuscrit, quel est cet objet dont s'occupe la linguistique et quel est son statut parmi les autres champs du savoir : 


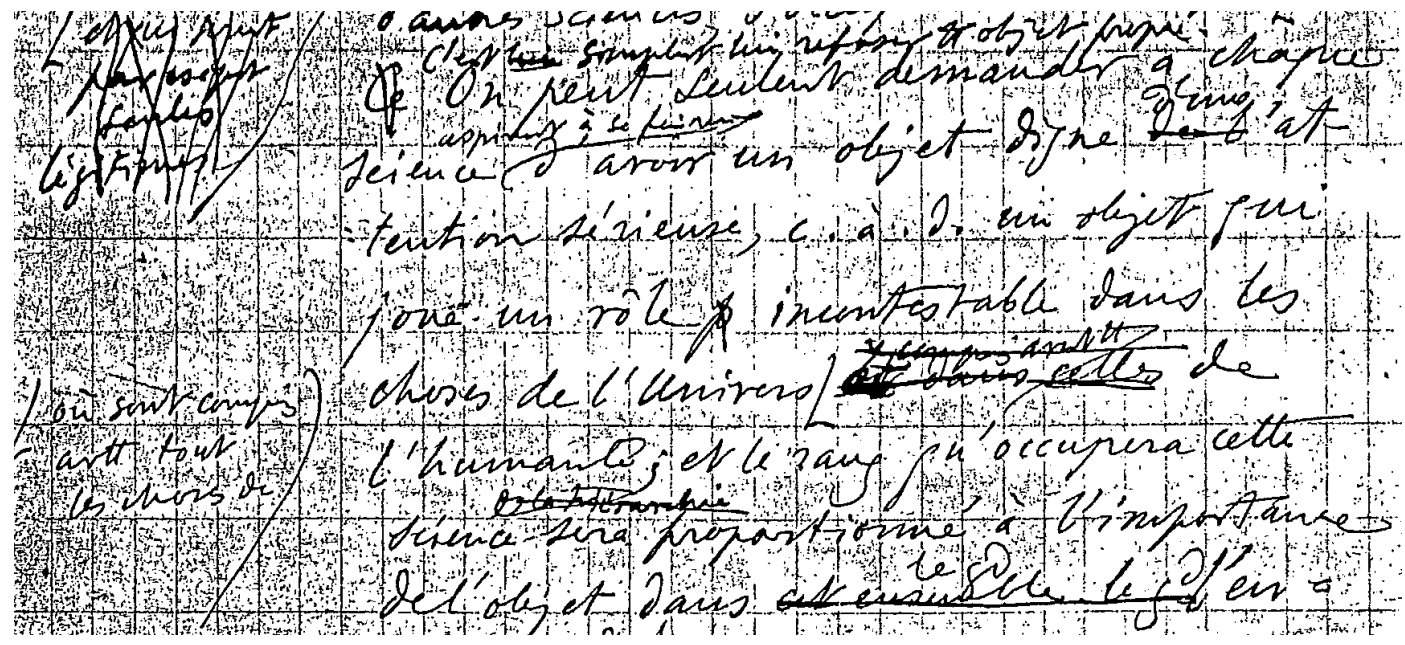

Enfin, l'objet d'une science ne peut pas être constitué à partir de son utilité pour les autres sciences.

Pour cela, il y a une claire détermination, après l'hésitation, pour parler de l'autonomie des études du langage et la possibilité du nouveau s'ouvre dans la page cinq: le terme utilité disparaît tandis que but et place réapparaissent et, but est associé, spécifiquement, au développement intérieur des études du langage:

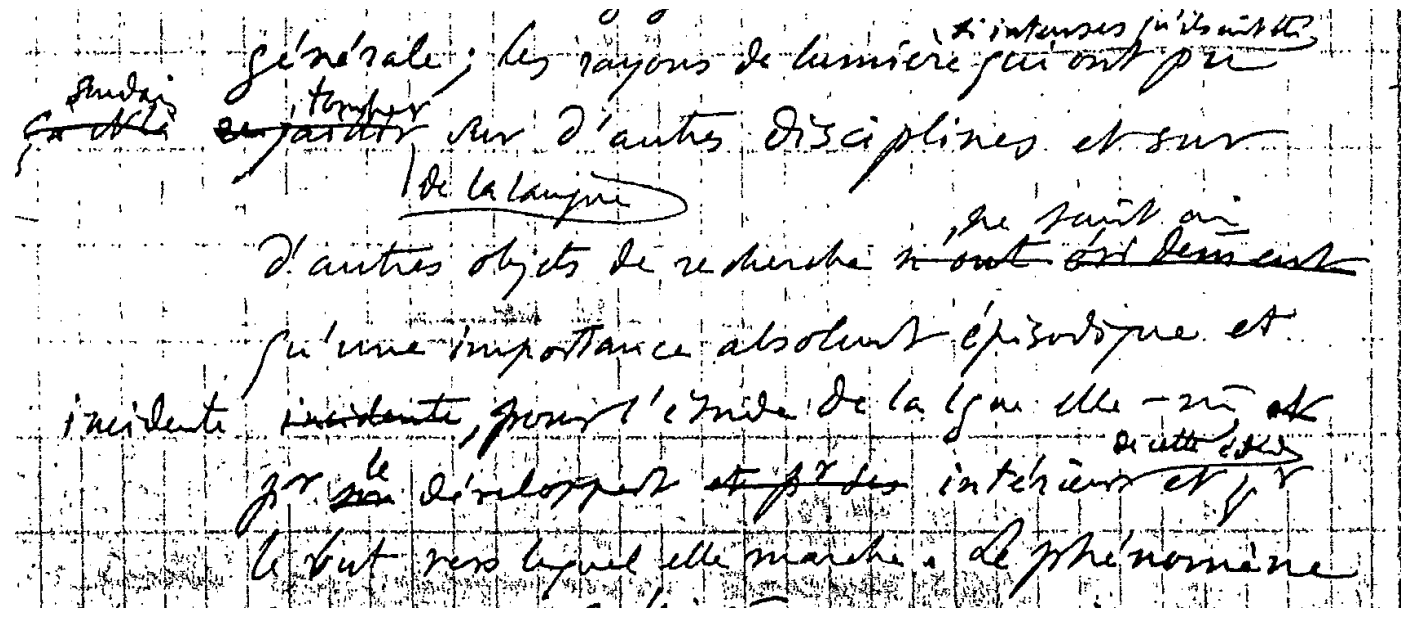

Parler sur le lieu de la linguistique par rapport aux autres sciences requiert de mettre une interrogation sur son objet et les observations de Saussure indiquent une direction dans laquelle son mouvement s'est produit; ces moments de tension dans son manuscrit, signalisés par ce premier groupe de ratures, montrent les impasses qui constituent l'acte d'écrire le nouveau.

\section{Deuxième groupe: entre le général et le particulier}

Ce deuxième groupe est composé par les termes 'général' et 'particulier', et ce qui le caractérise n'est pas seulement le fait que ces termes apparaissent raturés, mais la façon errante dont ils comparaissent dans le texte. En outre, ils fonctionnent comme le noyau_d'une constellation autour de laquelle gravitent 
d'autres termes qui maintiennent avec ce noyau des rapports sémantiques : individus, individuel ou espèce. Ou encore, des termes dérivés de ce noyau : généralisation, généralement ou particulièrement.

L'insistance avec laquelle ces termes se présentent en qualifiant des mots très variés tout au long du texte est particulière. Cette errance peut aussi se manifester par l'insistance avec laquelle quelques termes sémantiquement communs se répètent raturés et sans destin. Dans cet aspect, il est important d'observer ce qui arrive avec les termes présents dans une page très raturée, des termes qui, d'une certaine façon, sont alignés avec 'particulier' et 'général'. Ces termes sont: individuel(2d) et espèce(2e), rencontrés que dans la page sept, au moins trois fois chacun, en ratures ou en incises.

Mais c'est à la deuxième page des manuscrits, pendant que les questions sur lesquelles parle le premier groupe de ratures sont développées, que les termes particulièrement et individus surgissent raturés. Dans cette page, ces termes, bien que raturés, se font présents dans le but de caractériser le travail d'Adolf Pictet et, donc, n'ont pas de rapport direct et spécifique avec la linguistique et son objet. En plus de cela il est risqué de parier sur ce qui est écrit, effectivement, dans cet extrait du manuscrit. Il est important de souligner que l'errance qu'on a essayé de décrire au-dessus précède le point dans lequel ces termes vont trouver leur places. C'est-à-dire, avant ce point, il n'y a pas pour ces termes meilleur destin que les décombres d'une rature, la marginalité d'une incise ou d'une dérive incertaine.

Cependant, c'est à la cinquième page du manuscrit que l'on rencontre les motifs pour nommer particulier et général comme noyau du deuxième groupe. Initialement, il y a une référence à l'étude du langage dans la connaissance générale, encore dans le but de séparer la linguistique des autres sciences et, juste après, les termes 'général' et 'particulier' apparaissent clairement dans «lois générales » et «formes particulières » en faisant référence au langage. Accompagnons Saussure:

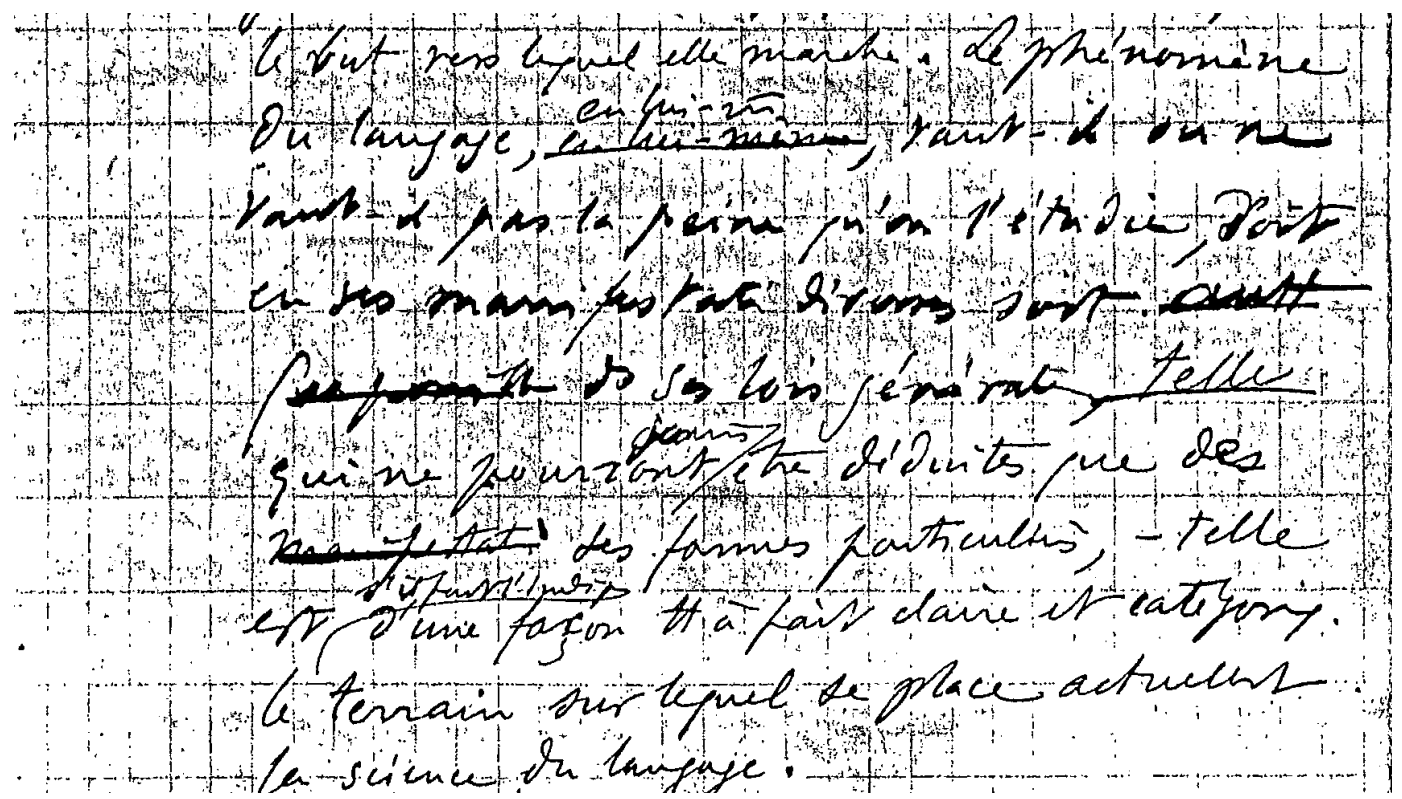

Langue et langage ne présentent pas de distinction précise et ne semblent pas être le plus important au moment où il faut indiquer de façon claire et catégorique le lieu dans lequel s'insère actuellement la science $d u$ langage. Soit quand il s'agit des manifestations diverses, soit quand il s'agit des lois générales, cette indication se soutient sur l'affirmation que l'étude du phénomène du langage ne peut pas renoncer à se tourner vers les formes particulières des langues. 
En considérant qu'un des aspects de l'étude descriptive est l'approche particulière de chaque langue, on peut voir dans ce manuscrit que le 'général' et le 'particulier', qui ont flotté jusqu'à un certain moment, finissent par atterrir et avec la fonction d'établir un rapport jusque-là inédit entre le général et le particulier dans les études du langage:

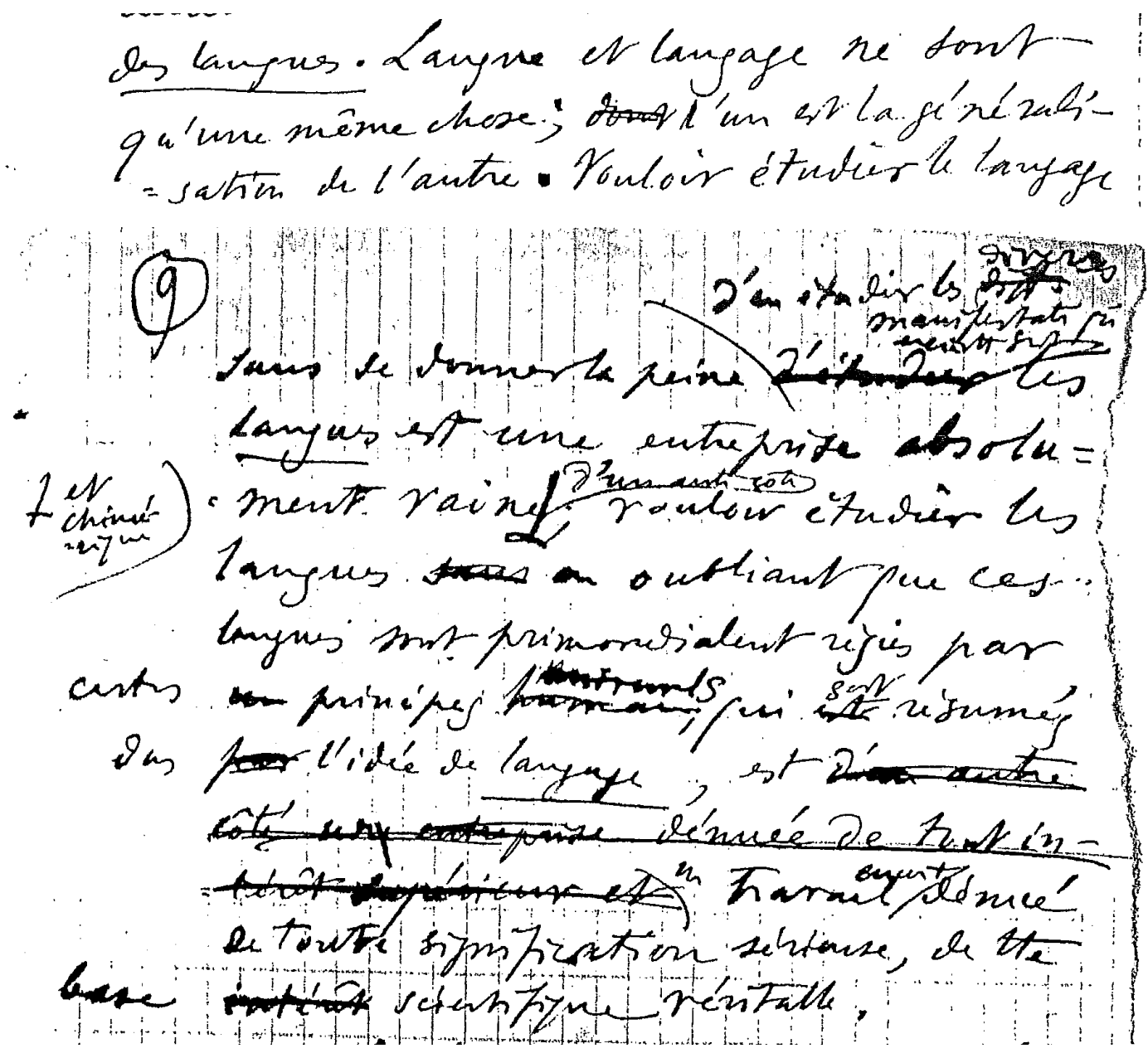

Il y a eu un mouvement dans les élaborations de Saussure. Cependant, on ne peut pas affirmer que celuici ait eu son dernier mot sur le sujet. Nous sommes intéressés de montrer la possibilité de saisir un mouvement que seul le processus de l'écriture par les ratures, les incises et l'errance nous permet de récupérer. En outre, ce mouvement qui accorde un certain lieu au général et au particulier dans les études du langage a de l'influence pour la définition de l'objet de la linguistique.

\section{Troisième groupe: l'objet de la linguistique}

Le dernier paragraphe de la page cinq des notes pour la Première Conférence est un extrait raturé, premièrement avec des traits horizontaux et ensuite avec des traits diagonaux. Dans ce paragraphe commençant par des phrases interrogatives, on rencontre les termes qui constituent le troisième groupe : langue, langage, parole ${ }^{4}$ : 


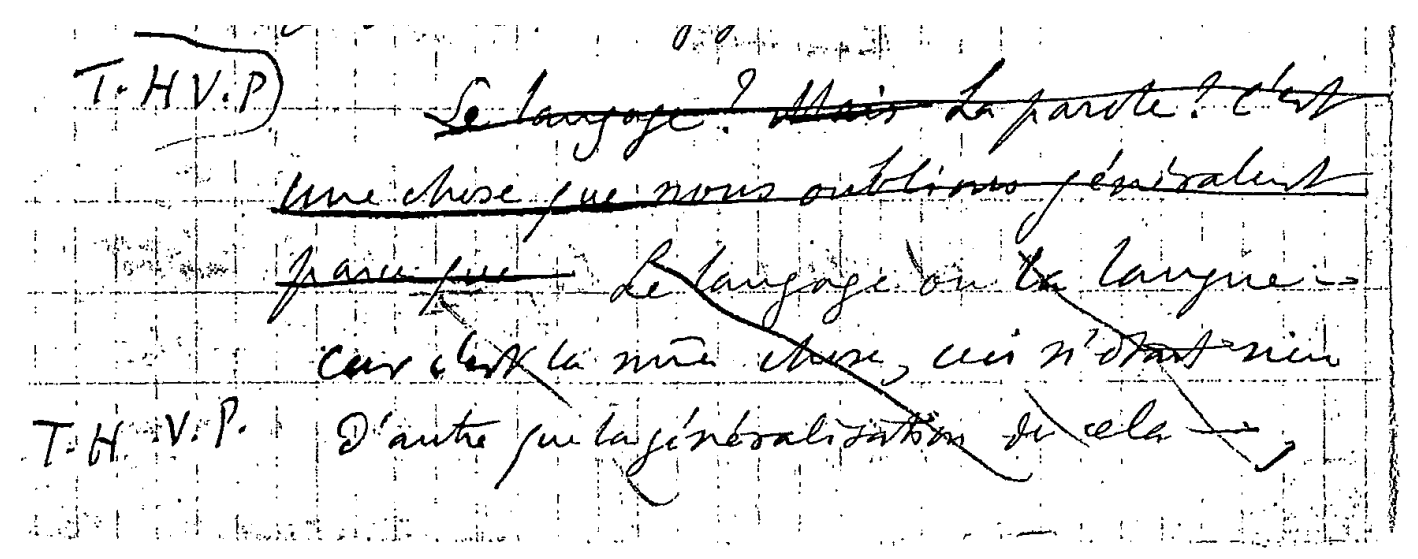

Donc, ces questions retombent sur : langage, parole et langue, c'est-à-dire, sur comment définir l'objet de la linguistique. Cette question traverse tout le manuscrit dont les ratures, les incises et l'errance révèlent la difficulté d'élaboration. Cette difficulté est présente dans l'interruption nécessaire du sens que ces mots possèdent dans le discours commun et le sens qu'ils doivent avoir dans la constitution de la science linguistique. C'est cela que l'on comprend lorsque l'on se tourne vers l'expression qui précède ces questions raturées : la science du langage.

On voit aussi, dans les trois dernières lignes raturées en diagonale, la première version de ce qui sera affirmé sans ratures dans la page huit: Langue et langage ne sont qu'une même chose; l'un est la généralisation de l'autre. Dans les pages suivantes, six et sept, le texte semble servir à séparer la nature du langage de la nature de l'homme pour que la première soit en conditions de solliciter son autonomie scientifique.

Dans ces pages du manuscrit, on lit une résistance à cet effacement et un travail qui a des effets sur la délimitation de cet objet. Ainsi, après les hésitations de la page sept dans laquelle les ratures et les incises sont plus nombreuses que dans le propre corps du texte qui n'est pas raturé, la page huit du manuscrit nous réserve une surprise. Langue et langage, par exemple, reviennent avec une affirmation catégorique semblable à celle sous les ratures dans la page cinq : Langue et langage ne sont qu'une même chose; l'un est la généralisation de l'autre. Mais il est important de mentionner que l'on a rencontré le mot des langues utilisé pour la première fois dans ces manuscrits et qu'il jouera un rôle relevant dans les explications de la question.

On a pensé qu'il y a dans ce manuscrit un rapprochement entre la question de l'objet de la linguistique et une position claire quant au besoin de le délimiter, bien qu'il n'y ait pas encore de distinctions plus précises, par exemple, entre langue, langage et parole. On sait que le rapport entre langue et langage souligné au-dessus ne sera pas conservé, comme on peut le lire dans le Cours de Linguistique Général. Cependant, dans ce manuscrit s'opère un déplacement vers l'ordre propre de la langue vu qu'il y a une tentative de destituer cet objet de ce qui est accessoire et de cerner ce qui est central, propre à lui-même.

Dans ce travail de séparer l'objet des études du langage de ce qui lui est proche mais non propre se dessine la possibilité de fonder un champ : la dénommée linguistique moderne. C'est cela que l'on peut conclure de ce jeu de Saussure entre des ratures et des élaborations présentes dans l'écriture de ce manuscrit.

\section{Quelques observations}

Il est nécessaire de souligner que les questions mises en évidence à partir des ratures dans ce manuscrit de Saussure - la discussion entre le général et le particulier, l'objet de la linguistique et le concept de la 
parole- ne sont pas déconnectées du travail de Saussure comme professeur ou conférencier, et non plus des élaborations présentes dans d'autres manuscrits et aussi peuvent être surprises dans le Cours de Linguistique Générale. C'est ce que nous traiterons succinctement, ci-après.

La question entre le général et le particulier que nous avons vue thématisée par Saussure semble avoir dans ce manuscrit à peine son début, De Mauro (1986) affirme que Saussure :

\begin{abstract}
(...)n'abandonne pas, après 1885 , les réflexions sur la théorie générale. Cette exigence, affirmée dans le compte rendu de Schimidt de 1897 (supra 351), est déjà justifiée lors des leçons inaugurales de 1891 dans lesquelles, comme nous avons dit (supra 354), il défend la nécessaire complémentarité des analyses particulières et de la théorie générale.(Grifo nosso; p.360)
\end{abstract}

Cette question ne pourrait pas être déconnectée de l'appréhension de l'objet de la Linguistique par Saussure, et à ce propos Benveniste affirme : «Certains linguistes se plaignent du plaisir que prend Saussure à souligner des paradoxes dans le fonctionnement du langage. Le langage, toutefois, est vraiment ce qu'il y a de plus paradoxal dans le monde, et malheureux sont ceux qui ne le voient pas » (1991:45)

La complexité de cet objet pour Saussure est aussi remarquée dans la lettre qu'il adresse, le 4 janvier 1894, à son ami Meillet :

Sans cesse l'ineptie absolue de la terminologie courante, la nécessité de la reformer, et de montrer pour cela quelle espèce d'objet est la langue en général, vient gâter mon plaisir historique, quoique je n'aie pas de plus cher vœu que de n'avoir pas à m’occuper de la langue en général.

Cela finira malgré moi par un livre où, sans enthousiasme ni passion, j'expliquerai pourquoi il n'y a pas un seul terme employé en linguistique auquel j'accorde un sens quelconque. Et ce n'est qu'après cela, je l'avoue, que je pourrai reprendre mon travail au point où je l'avais laissé. (Grifo nosso; De Mauro;1986:355)

Saussure montre des signes de malaise avec la «terminologie actuelle », cependant, on ne peut seulement modifier cette terminologie en montrant quelle espèce d'objet est la langue en général. Ainsi, il se propose de suspendre la linguistique qui se faisait jusqu'alors et de prendre soin des problèmes qui lui ont causé ce mal-être pour qu'il puisse retourner à son «plaisir historique ». Chemin sans retour. Cette suspension a ouvert la possibilité d'une linguistique à partir de laquelle le «plaisir historique »a été interdit vu que la structure (terme déduit des élaborations de Saussure) depuis qu'il lui fait de l'ombre.

Comme effet de ce difficile parcours de Saussure, le concept de parole nous parviendra comme l'un des plus controversés. Le Cours de Linguistique Générale l'apporte de manière négative, ou c'est-à-dire, à la construction du concept de langue, Saussure laisse surgir ce qui vient à être la parole alors que ce n'est pas la langue. La parole apparaît comme un excès de la langue. Saussure la place aussi comme secondaire dans les études linguistiques, elle ne serait pas capable d'occuper en elle-même la place de l'objet de la linguistique. Dans ce parcours théorique, présent dans le CLG, nous pouvons appréhender ce qui est la parole concernant la langue ainsi que les relations entre l'une et l'autre et, plus que cela, appréhender une réflexion sur la place de la langue dans la constitution de la linguistique. Les manuscrits Saussuriens, présents à la bibliothèque publique de Genève fournissent quelques informations importantes sur le processus d'écriture de Saussure, en particulier en ce qui concerne la construction de certains concepts, même si ce n'est pas toujours un processus qui nous permet de trouver le concept, en effet, construit, comme dans le cas de la parole.

Les thèmes apportés ici à partir de ce manuscrit ont déjà été visités par Saussure lui-même avant ce manuscrit et il ne les a pas oubliés après 1891. De telles préoccupations peuvent être repérées dans divers matériaux : dans des cours comme en témoignent les cahiers de ses élèves, dans des écrits théoriques ou des lettres adressées à des amis ou collègues. Quelques linguistes aussi, avant lui, se sont dédiés à ces questions ${ }^{5}$. De nombreux chercheurs ont souligné la pertinence de la discussion réalisée à ce propos au tournant des du $\mathrm{XIX}^{\mathrm{e}}$ au $\mathrm{XX}^{\mathrm{e}}$ siècles. Cette insistance nous a motivés pour aborder la question 
mais il convient de noter que ce que nous avons l'intention d'apporter en ce moment est la productivité des ratures dans ces écrits de Ferdinand de Saussure afin que nous puissions accompagner son mouvement dans lequel il a été, plus tard, reconnu comme fondement de la linguistique moderne, justement à partir de voies comme celles-ci et, en particulier, de ses effets dans l'architecture conceptuelle de Saussure.

Ainsi, ce que nous traiterons ensuite, reprendra ce qui a été présenté dans les manuscrits de Saussure afin de penser la rature comme une partie prenante de la fondation de la linguistique.

\section{Conclusion}

Il est important de noter que notre analyse, à partir des ratures, nous a permis d'indiquer l'une des directions dans lesquelles a eu lieu le mouvement de Saussure lors de la constitution de la linguistique, ainsi que les moments de tension dans ce mouvement, qui sont signalés dans le manuscrit analysé, à travers les groupes de ratures que nous avons proposés. Outre cela, il nous parait important d'indiquer l'impasse qui apparaît dans l'acte d'écrire le nouveau. Il est important de souligner que la trajectoire errante, que quelques termes ou phrases entières suivent dans la répétition et la rature, précède logiquement le point où ces termes vont trouver leur place. C'est-à-dire qu'avant ce point-là, il n'y a pas de meilleur destin pour ces termes que les décombres d'une rature, la marginalité d'un ajout ou la dérive incertaine. Ici, notre travail a déjà délimité ce 'point aveugle'. Ainsi, cette délimitation nous aidera à baliser le ton de ce travail et nous permettra d'avancer un peu plus et de penser à la puissance de ce 'point aveugle' dans le parcours saussurien.

Il faut cependant expliciter que, dans ce travail, l'expression 'point aveugle' pourrait être prise comme expression populaire puisque la seule possibilité qui nous est offerte est celle qui ne se restreint pas aux perspectives optiques, vu que dans ces perspectives le point aveugle est décelable, ce qui se perd de vue dans un point pouvant être vu depuis un autre point optique. Nous partons donc de la reconnaissance qu'il y a, pour le parlêtre, quelque chose qui lui échappe en définitif. Ce qui se dérobe lui cause, par conséquent, un malaise propre du non-sens, le destin donné à ce malaise devenant ainsi singulier.

Un point, quelque chose qui détient le développement d'une phrase en lui attribuant un lieu parmi d'autres, l'empêchant de continuer ad infinitum. Ce point de 'ça suffit' dans le glissement incessant du sens, élevé par Lacan à un élément déterminant de structure, est un point qui agit rétroactivement. C'est un point décisif pour le sens. Sa place n'est pas une place quelconque dans l'ensemble du langage et assume, semble-t-il, la fonction du signifiant: produire des effets de sens. Par contre, le terme qui suit dans l'expression 'point aveugle' nous amène vers une autre place, une place étanche où l'articulation propre du signifiant échoue, où le savoir échoue, où domine une présence du non-sens ; "aveugle" dans cette expression scelle un pacte avec le terme utilisé pour désigner ce qui appartient au domaine de "l'insoluble".

Dans le Séminaire 18, Lacan reprend son texte "L'instance de la lettre dans l'inconscient", lors de la réunion du 17 mars 1971, pour parler de ce qu'il avait déjà dit: 'une lettre arrive toujours à sa destination'. Mais cette fois-ci, il y ajoutera que : "La lettre qui arrive à son destin est la lettre qui arrive à la police qui ne comprend rien" (p.95). Donc, le destin de la lettre est de comparaître comme illisible. Ainsi, au-delà de notre recherche précédente, nous signalons maintenant le lieu de l'illisible.

Par conséquent ce que nous avons délimité auparavant, c'est-à-dire la détermination de ce qui constitue un 'point aveugle', peut être considéré à présent de la manière suivante :

1) ce qui ne s'éclaire pas: chez Saussure et chez plusieurs d'entre nous, l'obscur anagramme qui suscite des hypothèses qui vont du délire à la folie,

2) ce qui ne s'explique pas: pour la Linguistique, le paradoxal et gênant Cours de Linguistique Générale qui fait encore - presque un siècle plus tard - l'objet de vives discussions concernant aussi bien les conditions de son édition que les concepts qu'il contient; et 
3) ce qui ne se comprend pas: pour ceux qui se risquent à la lecture des manuscrits saussuriens et se laissent arrêter un moment - ou longuement - sur le bord de la falaise mise en place sur le texte par une rature.

Lacan nous dira, dans le séminaire 18, que s'il y a quelque chose que l'on ne comprend pas du tout, là c'est l'espoir absolu, c'est le signe que l'on a été touché par cela ; et heureusement que l'on n'a rien compris parce que seul peut se comprendre ce qui est déjà dans l'esprit.

Je me souviens ici des mots d'Amado Alonso, traducteur du Cours en espagnol, dans les premières lignes de sa préface à l' œuvre de Saussure: il vaut tant par ce qu'il a découvert - nous dit Alonso - que par ce qu'il nous a laissé à découvrir.

Ces points aveugles ont peut-être joué le rôle de cause du travail Saussure; ainsi que pour quelques-uns qui l'ont écouté et beaucoup qui l'ont lu. Après tout, les études de Saussure sur les anagrammes, point aveugle par excellence, ont résisté à sa compréhension et à celle des chercheurs, ce qui d'ailleurs offre toujours un point de scansion aux théories saussuriennes dans la linguistique.

Les ratures dans ses manuscrits déploient le manque de certitude de Saussure, condition nécessaire à vouloir savoir. Le manque d'option du lecteur des manuscrits devant les ratures impose à la fois la reconnaissance des limites de Saussure et l'ouverture de la possibilité de donner suite à la lecture à défaut de la lumière saussurienne.

Nous croyons donc que le point aveugle dans les ratures a conduit Saussure à poursuivre les démarches sur la langue. Le point aveugle dans ses études sur les anagrammes instaure une suspension sur tout ce que l'on voulait dire sur la langue, et les points aveugles dans le Cours bouleversent la linguistique.

À ce moment, il nous semble important de dire que le point aveugle est inéliminable par le 'parlêttre' et que, plus que cela, il peut être la condition de l'invention, une condition certes pas suffisante, mais nécessaire.

\section{Références bibliographiques}

Alonso A. (1945). Prologo a la edicion española in SAUSSURE, F. Curso de Lingüística Generale. Édition crítique de Tulio de Mauro. Buenos Aires: Editorial Losada.

Alves-Bezerra, W.(2010). Narrativa do corpo e da fala. In Sabático. O Estado de São Paulo, 21 août 2010. 6.

Chidichimo, A.(2009). Les premières leçons de Saussure à Genéve, 1891: textes, témoins, manuscrits. In Cahiers Ferdinand de Saussure Revue suisse de linguistique générale, no.62. Publié par le por Cercle Ferdinand de Saussure. Genève: Librairie Droz S.A. 257-276.

Godel, R. (1957) Les Sources manuscrites du cours de linguistique generale de Ferdinand de Saussure. Genève: Librairie Droz.

Lacan, J.(2009) O Seminário: de um discurso que não fosse semblante - livro 18. Texte établi par Jacques-Alain Miller. Traduction de Vera Ribeiro. Rio de Janeiro: Zahar.

Matsuzawa, Kazuhiro. (2006). Édition génétique de la première conférence à l'université de Genève (novembre 1891). In Texto!, vol.XI, n². Disponible sur http://www.revue-texto.net/1996-2007/Saussure/De_Saussure/ Conferences/ Matsuzawa_CG1.pdf. Consulté le 03/03/2010.

Normand, C. (2009). Entrevista. Revista Letras \& Letras, vol. 25 n.1. Uberlândia: Editora da Universidade Federal de Uberlândia. Disponible sur: http://www.letraseletras.ileel.ufu.br/viewissue.php?id=16 . Consulté le: 03/06/2010. 13-38.

Normand, C. (2006). Allegro ma non troppo - invitations à la linguistique. Ed.Ophrys. Paris.

Parret, H. 1993[4]. Les manuscrits saussuriens de Harvard. In Cahiers Ferdinand de Saussure, vol. 47, Publié par le Cercle Ferdinand de Saussure. Genève: Librairie Droz S.A. 179-234. 
Puech, C. (1998). L'esprit de Saussure. Paris contre Genève: l'héritage saussurien, Actes de la journée F. de Saussure. Modèles linguistiques. Tome XX, Fascicule 1 (S. Bouquet ed.) ; Presses Universitaires de Lille, 1998. p.79-93.

Saussure, F. de. (1973) Curso de Linguística Geral. [1916] Edité par Charles Bally \& Albert Sechehaye avec la collaboration d'Albert Riedlinger. Traduction A. Chelini, J. P. Paes e I. Blikstein. 5 a éd. São Paulo: Cultrix.

Saussure, F. de. (1891). 'Trois premières conférences'. In 'Papiers Ferdinand de Saussure', 3951: 'Notes de Linguistique Générale'. Bibliotèque de Genève.

Saussure, F. de. (2002). Écrits de Linguistique Générale; Texte établi et édité par Simon Bouquet et Rudolf Engler. Paris: Editions Gallimard.

Saussure, F. de. (1961). Lettres de Ferdinand de Saussure à Antoine Meillet - 1894-1911. In: Emile Benveniste (ed). Cahiers Ferdinand de Saussure Revue suisse de linguistique générale, N$^{\circ}$ 21: 89-135. Genève: Librairie Droz S.A..

Saussure, F. de. (1968-1989) Cours de linguistique générale: édition critique par Rudolf Engler. Tome 1, Otto Harrassowitz - Wiesbaden.

Saussure, F. de. (1974-1990). Cours de linguistique générale: édition critique par Rudolf Engler. Tome 2: appendice - Notes de F. de Saussure sur la linguistique générale, Otto Harrassowitz - Wiesbaden.

Silveira, E. (2007). As marcas do movimento de Saussure na fundação da linguística. Campinas-SP: Ed.Mercado de Letras/FAPESP.

Silveira, E. (2004) Revisitando uma das chamadas exclusões saussureanas: a história. In Revista de Estudos Linguísticos. Taubaté. 2004. Disponible sur www.gel.org.br/estudoslinguisticos/.../revisitando_chamadas.pdf Consulté le 12/05/2011.

Starobinski, J. (1971). As palavras sob as palavras : os anagramas de Ferdinand de Saussure. Traduction Carlos Vogt. São Paulo: Editora Perspectiva.

\footnotetext{
${ }^{1}$ Ce travail fait partie de la recherche "Le statut de la rature dans la fondation de la linguistique" financée par le CNPq, procédure $\mathrm{N}^{\circ}$ 309215/2008-0.

${ }^{2}$ Je remercie la Bibliothèque de Genève pour m'avoir mis à disposition les manuscrits de Ferdinand de Saussure ainsi que de m'avoir autorisé à faire des copies des 'Trois Conférences'.

${ }^{3}$ La délimitation initiale de ces groupes, ainsi qu'une première réflexion sur la rature dans ces manuscrits de Ferdinand de Saussure, ont été réalisées par nous dans un travail de plus grande amplitude sur la fondation de la linguistique, voir Silveira (2007).

${ }^{4}$ Il faut noter que le terme parole ne réapparaît pas dans ce manuscrit à moins d'être raturé quelques pages avant (devant) (p.6) et dans la page 10 sans rature mais suivi de plusieurs ratures. Cependant, la moindre mention de ce terme à côté de langue et langage est indicatif du travail qui s'est produit plus tard pour différencier langue et parole.

${ }^{5}$ Il ne faut pas oublier, ici, que les travaux en Grammaire comparée du XIX ${ }^{\mathrm{e}}$ siècle avaient déjà abordé de telles questions et certains auteurs (Whitney et Meillet en particulier) ont eu une grande influence sur le travail de Saussure.
} 